\title{
MAKING REASSIGNMENT ADJUSTABLE: THE LEVENBERG-MARQUARDT APPROACH
}

\author{
F. Auger $\quad$ E. Chassande-Mottin ${ }^{2} \quad$ P. Flandrin ${ }^{3}$ \\ ${ }^{1}$ LUNAM Université, IREENA, Saint-Nazaire, France. \\ ${ }^{2}$ Laboratoire AstroParticule et Cosmologie (APC), Université Paris Diderot, CNRS, CEA, Observatoire de Paris, France \\ ${ }^{3}$ Laboratoire de Physique de l'École Normale Supérieure de Lyon, CNRS and Université de Lyon, France \\ francois.auger@univ-nantes.fr, ecmeapc.univ-paris7.fr, patrick.flandrin@ens-lyon.fr
}

\begin{abstract}
This paper presents a new time-frequency reassignment process for the spectrogram, called the Levenberg-Marquardt reassignment. Compared to the classical one, this new reassignment process uses the second-order derivatives of the phase of the short-time Fourier transform, and provides the user with a setting parameter. This parameter allows him to produce either a weaker or a stronger localization of the signal components in the time-frequency plane.
\end{abstract}

Index Terms - nonstationary signals, time-frequency analysis, spectrogram, reassignment.

\section{INTRODUCTION}

In the recent years, a renewed interest appeared for the longstanding problem of designing strongly concentrated nonparametric time-frequency representations of nonstationary signals. The empirical mode decomposition [1] and the synchrosqueezing method $[2,3,4]$ are some of the most recently proposed solutions. The reassignment is maybe one of the oldest ones. The principle of this method is to move each value of the spectrogram from the point $(t, \omega)$ where it is computed to another point $\left(t^{\prime}, \omega^{\prime}\right)$ which is more representative of the localization of the signal energy. In [5, 6], this point was chosen as the centroid $(\hat{t}, \hat{\omega})$ of the signal energy in the neighborhood of $(t, \omega)$. As a consequence of this definition, there is no parameter allowing to adjust the concentration of the signal energy to the user's needs, so as to find a trade-off between sparsity and information loss. The aim of this paper is to present a new adjustable reassignment process, which allows the user to perform either a weak reassignment or a strong one. This process is defined and studied in $\$ 2$. Closed form expressions of this new reassignment are presented for several elementary signals and a Gaussian analysis window, and compared to the classical reassignment. A simple and reliable algorithm to compute the reassignment operators for any signal and any analysis window is provided in $\S 3$. Experimental results for a noisy signal are finally presented.

\section{DEFINITION AND PROPERTIES}

If $\mathrm{F}_{x}^{h}(t, \omega)$ denotes the short-time Fourier transform (STFT) of the signal $x$ using the analysis window $h, \mathrm{M}_{x}^{h}(t, \omega)$ its modulus and $\Phi_{x}^{h}(t, \omega)$ its phase, defined as

$$
\begin{aligned}
\mathrm{F}_{x}^{h}(t, \omega) & =\int_{-\infty}^{+\infty} x(u) h^{*}(t-u) e^{-\jmath \omega u} d u \\
& =\mathrm{M}_{x}^{h}(t, \omega) e^{\jmath \Phi_{x}^{h}(t, \omega)}
\end{aligned}
$$

then the classical reassignment operators can be derived from the partial derivatives of the phase of the STFT $[5,6]$ :

$$
\left(\begin{array}{c}
\hat{t}_{x}(t, \omega) \\
\hat{\omega}_{x}(t, \omega)
\end{array}\right)=\left(\begin{array}{c}
-\frac{\partial \Phi_{x}^{h}}{\partial \omega}(t, \omega) \\
\omega+\frac{\partial \Phi_{x}^{h}}{\partial t}(t, \omega)
\end{array}\right)
$$

To concentrate the signal energy, this reassignment process moves the spectrogram values towards the ridges [7] of the signal, which are the fixed points of the reassignment operators, $\hat{t}_{x}(t, \omega)=t$ and $\hat{\omega}_{x}(t, \omega)=\omega$, and therefore nullify the relative displacement

$$
R_{x}^{h}(t, \omega)=\left(\begin{array}{c}
t \\
\omega
\end{array}\right)-\left(\begin{array}{c}
\hat{t}_{x}(t, \omega) \\
\hat{\omega}_{x}(t, \omega)
\end{array}\right)=\left(\begin{array}{c}
t+\frac{\partial \Phi_{x}^{h}}{\partial \omega}(t, \omega) \\
-\frac{\partial \Phi_{x}^{h}}{\partial t}(t, \omega)
\end{array}\right)
$$

As a consequence, the reassignment can be considered as moving the signal energy from one step on the way to the closest ridge from the point $(t, \omega)$, i.e. to the closest point for which $R_{x}^{h}(t, \omega)=0$. This step can be deduced from numerical algorithms to find a root of $R_{x}^{h}(t, \omega)$. The classical reassignment operators can be considered as the first iteration of a fixed point algorithm, in which a sequence defined in the scalar case as $s_{n+1}=s_{n}-\lambda f\left(s_{n}\right)$, with $\lambda \in \mathbb{R}^{*}$ is hoped to converge to a root of $f(s)$. Eq. 4 can hence be written as

$$
\left(\begin{array}{c}
\hat{t}_{x}(t, \omega) \\
\hat{\omega}_{x}(t, \omega)
\end{array}\right)=\left(\begin{array}{c}
t \\
\omega
\end{array}\right)-R_{x}^{h}(t, \omega)
$$

This point of view allows to deduce other reassignment operators from other root finding algorithms. For example, the differential method, in which a differential equation $\frac{d s}{d u}=$ $-\lambda f(s(u))$ is simulated until reaching a steady state, leads to the differential reassignment defined in [8]. In the present paper, we suggest to design reassignment operators from the Levenberg-Marquardt (LM) algorithm [9, 10, 11] defined by the sequence $s_{n+1}=s_{n}-\frac{f\left(s_{n}\right)}{f^{\prime}\left(s_{n}\right)+\mu}$, with $\mu \in \mathbb{R}_{+}$. This algorithm is generally considered to be more robust than the Newton algorithm, obtained for $\mu=0$, because it converges even if the starting point is far from the final value. The re- 
assignment operators deduced from this algorithm will be defined as

$$
\begin{aligned}
\left(\begin{array}{c}
\tilde{t}_{x}(t, \omega) \\
\tilde{\omega}_{x}(t, \omega)
\end{array}\right) & =\left(\begin{array}{c}
t \\
\omega
\end{array}\right)-\left(\nabla R_{x}^{h}(t, \omega)+\mu I_{2}\right)^{-1} R_{x}^{h}(t, \omega)(6) \\
\nabla R_{x}^{h}(t, \omega) & =\left(\begin{array}{ll}
\frac{\partial R_{x}^{h}}{\partial t}(t, \omega) & \frac{\partial R_{x}^{h}}{\partial \omega}(t, \omega)
\end{array}\right) \\
& =\left(\begin{array}{cc}
1+\frac{\partial^{2} \Phi_{x}^{h}}{\partial t \partial \omega}(t, \omega) & \frac{\partial^{2} \Phi_{x}^{h}}{\partial \omega^{2}}(t, \omega) \\
-\frac{\partial^{2} \Phi_{x}^{h}}{\partial t^{2}}(t, \omega) & -\frac{\partial^{2} \Phi_{x}^{h}}{\partial t \partial \omega}(t, \omega)
\end{array}\right)
\end{aligned}
$$

where $\mu \in \mathbb{R}_{+}$and $I_{2}$ is the two-dimensional identity matrix. As for the classical reassignment operators, this reassignment process, which uses the second-order derivatives of the phase of the short-time Fourier transform [12], can be used to build a time-frequency distribution which will attempt to focus the signal energy around the signal ridges:

$$
\begin{aligned}
& \operatorname{LMR}_{x}^{h}(t, \omega) \\
& \quad=\iint\left|\mathrm{F}_{x}^{h}(u, \Omega)\right|^{2} \delta\left(t-\tilde{t}_{x}(u, \Omega)\right) \delta\left(\omega-\tilde{\omega}_{x}(u, \Omega)\right) d u \frac{d \Omega}{2 \pi}
\end{aligned}
$$

Although deduced from a purely mathematical justification, Eq. 8 satisfies the dimensional homogeneity. Denoting the physical unit of $x$ as $[x]$, then $[t]=s,[\omega]=s^{-1}$ and $\left[\Phi_{x}^{h}\right]=1$, therefore

$$
\begin{gathered}
{\left[\nabla R_{x}^{h}\right]=\left[\nabla R_{x}^{h}+\mu I_{2}\right]=\left[\left(\nabla R_{x}^{h}+\mu I_{2}\right)^{-1}\right]=\left(\begin{array}{ll}
1 & s^{2} \\
s^{-2} & 1
\end{array}\right),} \\
{\left[R_{x}^{h}\right]=\left[\left(\nabla R_{x}^{h}+\mu I_{2}\right)^{-1} R_{x}^{h}\right]=\left(\begin{array}{l}
s \\
s^{-1}
\end{array}\right)}
\end{gathered}
$$

and $\left[\operatorname{det}\left(\nabla R_{x}^{h}+\mu I_{2}\right)\right]=1$. The coefficients on the antidiagonal of $\left(\nabla R_{x}^{h}+\mu I_{2}\right)^{-1}$ have the required dimensionality for $R_{x}^{h}$ and $\left(\nabla R_{x}^{h}+\mu I_{2}\right)^{-1} R_{x}^{h}$ to have the same dimensionality.

This reassignment process also satisfies the time and frequency shift invariance:

$$
\begin{aligned}
\text { if } y(t) & =x\left(t-t_{0}\right) e^{\jmath \omega_{0} t} \\
\text { then } \mathrm{F}_{y}^{h}(t, \omega) & =\mathrm{F}_{x}^{h}\left(t-t_{0}, \omega-\omega_{0}\right) e^{-\jmath\left(\omega-\omega_{0}\right) t_{0}} \\
\Phi_{y}^{h}(t, \omega) & =\Phi_{x}^{h}\left(t-t_{0}, \omega-\omega_{0}\right)-\left(\omega-\omega_{0}\right) t_{0}, \\
\text { therefore } R_{y}^{h}(t, \omega) & =R_{x}^{h}\left(t-t_{0}, \omega-\omega_{0}\right) \\
\nabla R_{y}^{h}(t, \omega) & =\nabla R_{x}^{h}\left(t-t_{0}, \omega-\omega_{0}\right) \\
\text { hence } \tilde{t}_{y}(t, \omega) & =t_{0}+\tilde{t}_{x}\left(t-t_{0}, \omega-\omega_{0}\right) \\
\tilde{\omega}_{y}(t, \omega) & =\omega_{0}+\tilde{\omega}_{x}\left(t-t_{0}, \omega-\omega_{0}\right)
\end{aligned}
$$

This means that this process does not depend on the choice of the origin of the time and frequency plane, and therefore only produces a relative move from the point $(t, \omega)$.

Finally, it can be shown that the use of the second-order derivatives does not divert the energy of a monocomponent signal away from its desired localization: for a monocomponent signal $x(t)=|x(t)| e^{\varphi_{x}(t)}$ with a Fourier transform $X(\omega)=|X(\omega)| e^{\Psi_{x}(\omega)}$, it can be shown that when $h(t) \rightarrow$ $\delta(t), \Phi_{x}^{h} \rightarrow \varphi_{x}(t)-\omega t, \tilde{t}_{x} \rightarrow t$ and $\tilde{\omega}_{x} \rightarrow \frac{\mu \omega+\varphi_{x}^{\prime}(t)}{1+\mu}$, whereas when $h(t) \rightarrow 1, \Phi_{x}^{h} \rightarrow \Psi_{x}(\omega), \tilde{t}_{x} \rightarrow \frac{\mu t-\Psi_{x}^{\prime}(\omega)}{1+\mu}$ and $\tilde{\omega}_{x} \rightarrow \omega$. When $\mu \rightarrow+\infty$, the spectrogram is left unchanged, whereas when $\mu \rightarrow 0$, the whole energy is correctly localised on the instantaneous frequency curve $\varphi_{x}^{\prime}(t)$ when $h(t) \rightarrow \delta(t)$ and on the group delay curve $-\Psi_{x}^{\prime}(\omega)$ when $h(t) \rightarrow 1$.

\section{SOME ANALYTICAL EXAMPLES}

To evaluate the interest of this new reassignment process, one may consider the case of some simple elementary signals, for which closed form expressions of $\tilde{t}_{x}$ and $\tilde{\omega}_{x}$ can be derived and compared to the classical reassignment operators. In this section, we will use a unit energy Gaussian window of time width $\lambda, h(t)=\lambda^{-1 / 2} \pi^{-1 / 4} e^{-t^{2} /\left(2 \lambda^{2}\right)}$.

\subsection{Sinusoid}

For a complex sinusoid of angular frequency $\omega_{0}, x(t)=$ $e^{\jmath \omega_{0} t}$, we have [13] $\Phi_{x}^{h}(t, \omega)=\left(\omega_{0}-\omega\right) t$, hence

$$
\begin{aligned}
\frac{\partial \Phi_{x}^{h}}{\partial \omega}(t, \omega) & =-t, \quad \frac{\partial \Phi_{x}^{h}}{\partial t}(t, \omega)=\omega_{0}-\omega \\
\frac{\partial^{2} \Phi_{x}^{h}}{\partial t \partial \omega}(t, \omega) & =-1, \frac{\partial^{2} \Phi_{x}^{h}}{\partial t^{2}}(t, \omega)=0 \text { and } \frac{\partial^{2} \Phi_{x}^{h}}{\partial \omega^{2}}(t, \omega)=0 .
\end{aligned}
$$

The classical reassignment operators, $\hat{t}_{x}(t, \omega)=t$ and $\hat{\omega}_{x}(t, \omega)=\omega_{0}$, force all the signal energy to lie on the straight line $\omega=\omega_{0}$. With the LM reassignment, $\tilde{t}_{x}(t, \omega)=t$ and $\tilde{\omega}_{x}(t, \omega)=\frac{\omega_{0}+\mu \omega}{1+\mu}$. There is no time displacement and $\tilde{\omega}_{x}$ is an angular frequency between $\omega_{0}$ (obtained for $\mu=0$ ) and $\omega$ (obtained for $\mu \rightarrow+\infty$ ). This process will focus the signal around the straight line $\omega=\omega_{0}$, since $\left|\tilde{\omega}_{x}(t, \omega)-\omega_{0}\right|<\left|\omega-\omega_{0}\right|$, with a degree of concentration that can be chosen by the user through the parameter $\mu$.

\subsection{Impulse}

For an impulse localised at $t_{0}, x(t)=\delta\left(t-t_{0}\right)$, we have [13] $\Phi_{x}^{h}(t, \omega)=-\omega t_{0}$, hence

$$
\begin{aligned}
\frac{\partial \Phi_{x}^{h}}{\partial \omega}(t, \omega) & =-t_{0}, \quad \frac{\partial \Phi_{x}^{h}}{\partial t}(t, \omega)=0 \\
\frac{\partial^{2} \Phi_{x}^{h}}{\partial t \partial \omega}(t, \omega) & =0, \frac{\partial^{2} \Phi_{x}^{h}}{\partial t^{2}}(t, \omega)=0 \text { and } \frac{\partial^{2} \Phi_{x}^{h}}{\partial \omega^{2}}(t, \omega)=0 .
\end{aligned}
$$

Here also, the classical reassignment operators, $\hat{t}_{x}(t, \omega)=t_{0}$ and $\hat{\omega}_{x}(t, \omega)=\omega$, move all the signal energy on the straight line donc la totalité de l'énergie du signal $t=t_{0}$. With the LM reassignment, $\tilde{t}_{x}(t, \omega)=\frac{t_{0}+\mu t}{1+\mu}$ and $\tilde{\omega}_{x}(t, \omega)=\omega$. As in the previous case, $\left|\tilde{t}_{x}(t, \omega)-t_{0}\right|<\left|t-t_{0}\right|$, and the user can choose the increase of concentration as desired through the choice of a value of $\mu$ between 0 (leading to a maximal concentration since $\tilde{t}_{x}(t, \omega)=t_{0}$ ) and $+\infty$ (providing no concentration increase since $\tilde{t}_{x}(t, \omega)=t$ ).

\subsection{Gaussian logon}

For $x(t)=T^{-1 / 2} \pi^{-1 / 4} e^{-t^{2} /\left(2 T^{2}\right)}$, i.e. for a Gaussian logon centered at the origin of the time-frequency plane and of time width $T$, we have [13] $\Phi_{x}^{h}(t, \omega)=-\frac{T^{2}}{\lambda^{2}+T^{2}} \omega t$, hence

$$
\begin{aligned}
\frac{\partial \Phi_{x}^{h}}{\partial \omega}(t, \omega) & =-\frac{T^{2} t}{\lambda^{2}+T^{2}}, \quad \frac{\partial \Phi_{x}^{h}}{\partial t}(t, \omega)=-\frac{T^{2} \omega}{\lambda^{2}+T^{2}} \\
\frac{\partial^{2} \Phi_{x}^{h}}{\partial t \partial \omega}(t, \omega) & =-\frac{T^{2}}{\lambda^{2}+T^{2}}, \frac{\partial^{2} \Phi_{x}^{h}}{\partial t^{2}}(t, \omega)=\frac{\partial^{2} \Phi_{x}^{h}}{\partial \omega^{2}}(t, \omega)=0 .
\end{aligned}
$$


The classical reassignment causes the energy to be closer to the logon center: $\hat{t}_{x}(t, \omega)=\frac{T^{2}}{\lambda^{2}+T^{2}} t$ and $\hat{\omega}_{x}(t, \omega)=$ $\frac{\lambda^{2}}{\lambda^{2}+T^{2}} \omega$, hence $\left|\hat{t}_{x}\right|<|t|$ and $\left|\hat{\omega}_{x}\right|<|\omega|$. But the movements on both axes are antagonistic: if $\lambda \rightarrow 0, \hat{\omega}_{x} \rightarrow 0$ but $\hat{t}_{x} \rightarrow t$, whereas when $\lambda \rightarrow+\infty, \hat{t}_{x} \rightarrow 0$ but $\hat{\omega}_{x} \rightarrow \omega$. With the LM reassignment, $\frac{\tilde{t}_{x}(t, \omega)}{t}=\frac{\tilde{\omega}_{x}(t, \omega)}{\omega}=\frac{\mu\left(\lambda^{2}+T^{2}\right)}{\lambda^{2}+\mu\left(\lambda^{2}+T^{2}\right)}$. The point $\left(\tilde{t}_{x}, \tilde{\omega}_{x}\right)$ belongs to the line segment between the logon center $(0,0)$ (obtained for $\mu=0$ ) and the starting point $(t, \omega)$ (obtained for $\mu \rightarrow+\infty$ ). By an appropriate choice of the damping parameter $\mu$, the user can either weakly increase the signal localization provided by the spectrogram or to localise the whole energy at the point $(0,0)$ (whatever the time width $T$ ), something that the classical reassignment does not allow. This is a major difference between the LM reassignment and a more simple adjustable reassignment defined as $t^{\prime}=(1-\mu) t+\mu \hat{t}_{x}$ and $\omega^{\prime}=(1-\mu) \omega+\mu \hat{\omega}_{x}$ (with $0 \leq \mu \leq 1)$, that would not provide such a concentration increase. One may also notice that the point $\left(\hat{t}_{x}, \hat{\omega}_{x}\right)$ does not belong to the set of all the points $\left(\tilde{t}_{x}, \tilde{\omega}_{x}\right)$ obtained for $\mu \in \mathbb{R}_{+}$.

\subsection{Linear chirp}

For a constant amplitude linear chirp, $x(t)=e^{\jmath \alpha t^{2} / 2}$, we have [13] $\Phi_{x}^{h}(t, \omega)=\frac{\alpha t^{2}}{2}-\omega t-\frac{\alpha \lambda^{4}(\omega-\alpha t)^{2}}{2\left(1+\alpha^{2} \lambda^{4}\right)}$, hence

$$
\begin{aligned}
\frac{\partial \Phi_{x}^{h}}{\partial \omega}(t, \omega) & =-\frac{t+\alpha \lambda^{4} \omega}{1+\alpha^{2} \lambda^{4}}, \quad \frac{\partial \Phi_{x}^{h}}{\partial t}(t, \omega)=-\frac{\omega-\alpha t}{1+\alpha^{2} \lambda^{4}} \\
\frac{\partial^{2} \Phi_{x}^{h}}{\partial t \partial \omega}(t, \omega) & =-\frac{1}{1+\alpha^{2} \lambda^{4}}, \frac{\partial^{2} \Phi_{x}^{h}}{\partial t^{2}}(t, \omega)=\frac{\alpha}{1+\alpha^{2} \lambda^{4}} \\
\text { and } \frac{\partial^{2} \Phi_{x}^{h}}{\partial \omega^{2}}(t, \omega) & =-\frac{\alpha \lambda^{4}}{1+\alpha^{2} \lambda^{4}} .
\end{aligned}
$$

With the classical reassignment, $\hat{t}_{x}(t, \omega)=\frac{t+\alpha \lambda^{4} \omega}{1+\alpha^{2} \lambda^{4}}$ and $\hat{\omega}_{x}(t, \omega)=\alpha \hat{t}_{x}(t, \omega)$, hence the whole signal energy is localized on the straight line $\omega=\alpha t$. With the LM reassignment, $\tilde{t}_{x}(t, \omega)=t+\frac{\alpha \lambda^{4}}{(1+\mu)\left(1+\alpha^{2} \lambda^{4}\right)}(\omega-\alpha t)$ and $\tilde{\omega}_{x}(t, \omega)=$ $\omega-\frac{1}{(1+\mu)\left(1+\alpha^{2} \lambda^{4}\right)}(\omega-\alpha t)$. Since $\tilde{\omega}_{x}(t, \omega)-\alpha \tilde{t}_{x}(t, \omega)=$ $\frac{\mu}{1+\mu}(\omega-\alpha t)$, one can show that the point $\left(\tilde{t}_{x}, \tilde{\omega}_{x}\right)$ is always closer to the instantaneous frequency curve than the point $(t, \omega)$, is exactly on this curve when $\mu \rightarrow 0$ and is equal to $(t, \omega)$ when $\mu \rightarrow+\infty$. It should be noticed that here also the point $\left(\hat{t}_{x}, \hat{\omega}_{x}\right)$ does not belong to the set of all the points $\left(\tilde{t}_{x}, \tilde{\omega}_{x}\right)$ obtained for $\mu \in \mathbb{R}_{+}$.

To conclude these collection of closed form expressions of the reassigment operators, it should be noticed that the expressions of $\tilde{t}_{x}$ and $\tilde{\omega}_{x}$ lead to indeterminate expressions when $\mu \rightarrow 0$, since in these cases $\nabla R_{x}^{h}+\mu I_{2}$ is singular.

\section{EFFECTIVE IMPLEMENTATION}

The LM reassignment operators can not be computed only for a few simple analytic signals. On the opposite they can be computed reliably for any signal, thanks to the possibility to deduce the partial derivatives of $\Phi_{x}^{h}(t, \omega)$ from additional short-time Fourier transforms using particular analysis win- dows:

$$
\begin{aligned}
\frac{\partial \Phi_{x}^{h}}{\partial \omega}(t, \omega) & =-t+\operatorname{Re}\left(\frac{\mathrm{F}_{x}^{T h}(t, \omega)}{\mathrm{F}_{x}^{h}(t, \omega)}\right) \\
\frac{\partial \Phi_{x}^{h}}{\partial t}(t, \omega) & =\operatorname{Im}\left(\frac{\mathrm{F}_{x}^{D h}(t, \omega)}{\mathrm{F}_{x}^{h}(t, \omega)}\right) \\
\frac{\partial^{2} \Phi_{x}^{h}}{\partial t^{2}}(t, \omega) & =\operatorname{Im}\left(\frac{\mathrm{F}_{x}^{D^{2} h}(t, \omega)}{\mathrm{F}_{x}^{h}(t, \omega)}-\left(\frac{\mathrm{F}_{x}^{D h}(t, \omega)}{\mathrm{F}_{x}^{h}(t, \omega)}\right)^{2}\right)
\end{aligned}
$$

$$
\begin{aligned}
\frac{\partial^{2} \Phi_{x}^{h}}{\partial t \partial \omega}(t, \omega) & =\operatorname{Re}\left(\frac{\mathrm{F}_{x}^{T D h}(t, \omega)}{\mathrm{F}_{x}^{h}(t, \omega)}-\frac{\mathrm{F}_{x}^{T h}(t, \omega)}{\mathrm{F}_{x}^{h}(t, \omega)} \frac{\mathrm{F}_{x}^{D h}(t, \omega)}{\mathrm{F}_{x}^{h}(t, \omega)}\right) \\
\frac{\partial^{2} \Phi_{x}^{h}}{\partial \omega^{2}}(t, \omega) & =-\operatorname{Im}\left(\frac{\mathrm{F}_{x}^{T^{2} h}(t, \omega)}{\mathrm{F}_{x}^{h}(t, \omega)}-\left(\frac{\mathrm{F}_{x}^{T h}(t, \omega)}{\mathrm{F}_{x}^{h}(t, \omega)}\right)^{2}\right)
\end{aligned}
$$

with $T h(t)=t h(t), T^{2} h(t)=t^{2} h(t), D h(t)=\frac{d h}{d t}(t)$, $D^{2} h(t)=\frac{d^{2} h}{d t^{2}}(t), T D h(t)=t \frac{d h}{d t}(t), \operatorname{Re}(z), \operatorname{Im}(z)$ being respectively the real and imaginary part of the complex number $z$. Compared to the classical reassignment, the LM reassignment requires only three additional STFTs using the analysis windows $T D h, T^{2} h$ et $D^{2} h$. This computational cost is significantly reduced if, as in the previous section, $h(t)$ is a unit energy Gaussian window. In this case, $D h(t)=-T h(t) / \lambda^{2}, T D h(t)=-T^{2} h(t) / \lambda^{2}$, $D^{2} h(t)=-h(t) / \lambda^{2}+T^{2} h(t) / \lambda^{4}$ and expressions (17), (18) and (19) become

$$
\begin{aligned}
\frac{\partial \Phi_{x}^{h}}{\partial t}(t, \omega) & =-\frac{1}{\lambda^{2}} \operatorname{Im}\left(\frac{\mathrm{F}_{x}^{T h}(t, \omega)}{\mathrm{F}_{x}^{h}(t, \omega)}\right) \\
\frac{\partial^{2} \Phi_{x}^{h}}{\partial t^{2}}(t, \omega) & =-\frac{1}{\lambda^{4}} \frac{\partial^{2} \Phi_{x}^{h}}{\partial \omega^{2}}(t, \omega) \\
\frac{\partial^{2} \Phi_{x}^{h}}{\partial t \partial \omega}(t, \omega) & =-\frac{1}{\lambda^{2}} \operatorname{Re}\left(\frac{\mathrm{F}_{x}^{T^{2} h}(t, \omega)}{\mathrm{F}_{x}^{h}(t, \omega)}-\left(\frac{\mathrm{F}_{x}^{T h}(t, \omega)}{\mathrm{F}_{x}^{h}(t, \omega)}\right)^{2}\right)
\end{aligned}
$$

Compared to the classical reassignment, the LM reassignment then requires only an additional STFT using the analysis window $T^{2} h$. It should be also noticed that eq. (21) allows to write a surprising expression between the second order derivatives of the phase of the STFT (whatever the signal):

$$
\lambda^{2} \frac{\partial^{2} \Phi_{x}^{h}}{\partial t^{2}}(t, \omega)+\frac{1}{\lambda^{2}} \frac{\partial^{2} \Phi_{x}^{h}}{\partial \omega^{2}}(t, \omega)=0
$$

This relationship can be explained by the fact that for a Gaussian analysis window the two first-order derivatives and the three second-order derivatives are all deduced from the two complex numbers $\mathrm{F}_{x}^{T^{2} h} / \mathrm{F}_{x}^{T h}$ and $\mathrm{F}_{x}^{T h} / \mathrm{F}_{x}^{h}$, which necessarily involves some relationships between them. Eq. (22) seems to be new and complements previous results presented in [8], obtained when $\lambda=1$. This result will be developed further elsewhere [14].

\section{A NUMERICAL ILLUSTRATION}

The expressions presented in the previous section allow reliable computations of the LM reassignment operators for any signal. To illustrate this article, we choosed a 256-samples signal made of four deterministic components blurred with additive white Gaussian noise whith an SNR of $6 \mathrm{~dB}$. The 
chosen analysis window is a Gaussian window with $\lambda / T_{s}=$ $11, T_{s}$ being the sampling period. Fig. 1 shows the spectrogram reassigned with the classical operators. Fig. 2 shows a set of 20 representations obtained with the LM reassignment operators computed for $\mu$ between $10^{-2}$ and 40 . This figure shows that the new reassignment operators allow either a stronger concentration of the signal components than the classical ones or a weaker concentration, close to the nonreassigned spectrogram.

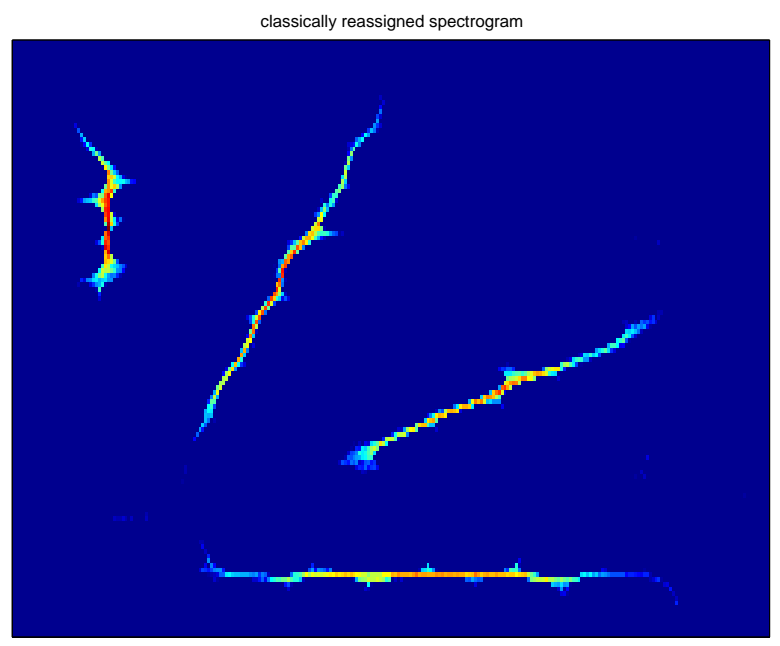

Fig. 1. Spectrogram of the illustrative signal obtained with the classical reassignment operators.

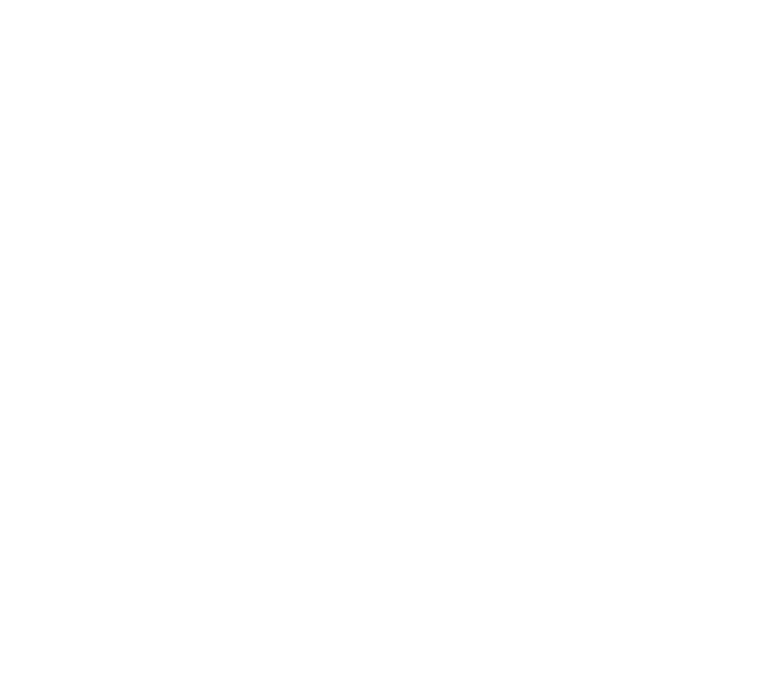

Fig. 2. Spectrogram of the illustrative signal obtained with the LM reassignment operators.

\section{CONCLUSION}

In this article, a new reassignment process has been defined, studied and illustrated. For the user, the main characteristic of this new tool is the possibility to fit it to his needs, either to strongly concentrate the signal energy or to mildly concentrate it, to preserve the reversibility of the distribution.
Among the possible future extensions of this work, one may first consider to associate it to a signal detection algorithm, to reassign strongly the deterministic components of a signal and only weakly the noise areas. It should also be possible to extend this reassignment process to continuous wavelet transforms and to all the Cohen's class members of bilinear timefrequency distributions. Other root finding principles could also be used to derive new reassignment operators. To reproduce and to deepen the results presented here, some MATLAB codes can be obtained from the first author upon request.

\section{REFERENCES}

[1] N.E. Huang, Z. Shen, S.R. Long, M.L. Wu, H.H. Shih, Q. Zheng, N.C. Yen, C.C. Tung, and H.H. Liu, "The empirical mode decomposition and Hilbert spectrum for nonlinear and non-stationary time series analysis," Proc. Roy. Soc. London A, vol. 454, pp. 903-995, 1998.

[2] E. Brevdo, H.T. Wu, G. Thakur, and N.S. Fuckar, "Synchrosqueezing and its applications in the analysis of signals with time-varying spectrum," Proceedings of the National Academy of Sciences of the United States of America, 2011, submitted.

[3] J. Lu, I. Daubechies, and H.T. Wu, "Synchrosqueezed wavelet transforms: an empirical mode decomposition-like tool," Appl. Comp. Harmonic Anal., , no. 30, pp. 243-261, 2011.

[4] H.T. Wu and I. Flandrin, P. andDaubechies, "One or two frequencies? the synchrosqueezing answers," Adv. Adapt. Data Anal., vol. 3, no. 1 \& 2, pp. 29-39, 2011.

[5] K. Kodera, C. de Villedary, and R. Gendrin, "A new method for the numerical analysis of nonstationary signals," Phys. Earth and Plan. Int., vol. 12, pp. 142-150, 1976.

[6] K. Kodera, R. Gendrin, and C. de Villedary, "Analysis of timevarying signals with small BT values," IEEE Transactions on ASSP, vol. 26, no. 1, pp. 64-76, Feb. 1978.

[7] N. Delprat, B. Escudié, P. Guillemain, R. Kronland-Martinet, P. Tchamitchian, and B. Torresani, "Asymptotic wavelet and Gabor analysis: extraction of instantaneous frequencies," IEEE Trans. on Information Theory, vol. 38, no. 2, pp. 644-664, 1992.

[8] E. Chassande-Mottin, I. Daubechies, F. Auger, and P. Flandrin, "Differential reassignment," IEEE Signal Processing Letters, vol. 4, no. 10, pp. 293-294, Oct. 1997.

[9] K. Levenberg, "A method for the solution of certain problems in least squares," Quart. Appl. Math., vol. 2, pp. 164-168, 1944.

[10] D. Marquardt, "An algorithm for least squares estimation of nonlinear parameters," SIAM J. Appl. Math., vol. 11, no. 2, pp. 431-441, 1963.

[11] P.E. Gill, W. Murray, and M.H. Wright, Practical optimization, Academic Press, 1982.

[12] D.J. Nelson, "Instantaneous higher order phase derivatives," Digital Signal Processing, vol. 12, pp. 416-428, 2002.

[13] E. Chassande-Mottin, F. Auger, and P. Flandrin, "Time frequency/time-scale reassignment," in Wavelets and signal processing, L. Debnath, Ed., pp. 233-268. Birkhauser, 2003.

[14] F. Auger, E. Chassande-Mottin, and P. Flandrin, "First- and second-order derivatives of the modulus and phase of the shorttime Fourier transform: Some new relations and applications," in preparation. 